\title{
Increased colorectal neoplasia in chronic ulcerative colitis complicated by primary sclerosing cholangitis: fact or fiction?
}

\begin{abstract}
Summary
It is well accepted that patients with ulcerative colitis (UC) are at increased risk of developing colorectal carcinoma. Since 1992, several studies have examined the hypothesis that patients with concomitant primary sclerosing cholangitis (PSC) are at significantly increased risk of developing colorectal cancer or dysplasia. The size, design, end points, and populations involved in these studies have varied but critical review suggests that colorectal cancer is more common in the setting of PSC. Although the data do not allow exact quantification of the increased relative risk, there are nevertheless implications, both for understanding disease pathogenesis and for clinical practice.
\end{abstract}

\section{Introduction}

An increased risk of developing colorectal carcinoma in patients with UC was first recognised in the 1920s. ${ }^{1}$ The magnitude of this risk varies in different studies with a range in cumulative risk after diagnosis in patients with pancolitis of between $1 \%$ and $3 \%$ at 10 years, $10 \%$ at 20 years, and $25 \%$ at 35 years. ${ }^{2-5}$ It is now widely accepted that risk factors for malignant transformation are duration of disease and extent of colitis. The presence of graded dysplasia $^{6}$ or DNA aneuploidy within the colorectal mucosa, and an early age at onset have been described as additional risk factors. ${ }^{4}$ It has also been suggested that pharmacological therapy of colitis with sulphasalazine or mesalazine may be associated with a reduced incidence of neoplastic transformation. ${ }^{78}$

PSC is a disease of unknown aetiology characterised by cholestasis associated with diffuse inflammation and fibrosis of the entire biliary tract. ${ }^{9}$ PSC has a variable clinical course, progressing eventually to cirrhosis and premature death from hepatic failure. Furthermore, PSC is accompanied by a risk of developing carcinoma of the bile ducts. ${ }^{10}$

Although PSC may occur in isolation, it is closely associated with inflammatory bowel disease, in particular UC. Up to $80 \%$ of patients with PSC also have UC, usually with extensive or total colonic involvement. ${ }^{11}$ The prevalence of PSC in UC is much lower, with its occurrence related to the extent of disease. A population based study found a prevalence of $5.5 \%$ in patients with substantial colitis and of $0.5 \%$ in patients with distal colitis only, ${ }^{12}$ although these values are likely to underestimate the risk of PSC as not all patients with PSC have abnormal liver function tests. ${ }^{13}$

The concept that PSC is associated with an increased risk of colorectal neoplasia in patients with UC was proposed by Broomé et al in 1992. ${ }^{14}$ In a study of 17 patients with UC who were found to have dysplasia, carcinoma, and/or DNA aneuploidy, 28\% had coexistent PSC. This led to the hypothesis that PSC was an independent risk factor for the development of colorectal neoplasia in patients with existing UC.

This hypothesis has remained a topic of extensive debate within the medical literature. ${ }^{15}$ This review aims to evaluate the available evidence to date, debate the possible mechanisms that may underlie association, and finally discuss the possible impact of any findings on current and future clinical practice.

\section{Clinical studies}

Since 1992, several studies have examined the role of PSC in the development of colorectal neoplasia in the setting of chronic UC. The studies are summarised in table 1 . The investigative format of the studies evolved with time, with the early focus being on the prevalence of PSC in UC complicated by neoplasia, and later on follow up of patients with UC with or without coexistent PSC.

Of these studies, eight have concluded that the risk of colorectal neoplasia in UC is greater in patients with PSC. ${ }^{14}{ }^{16-22}$ Only two studies (both from the Mayo Clinic but using different patients) have definitely concluded that there is no increased colorectal cancer risk in PSC. ${ }^{23}{ }^{24}$ Two further studies do not allow definite conclusions-one because of small numbers and the other due to variable end points. ${ }^{25} 26$

\section{POSITIVE STUDIES}

Following the initial paper by Broomé and colleagues, ${ }^{14}$ the association between PSC, dysplasia, aneuploidy, and colorectal cancer was re-examined in a cohort of 79 patients with extensive and chronic colitis enrolled in the Denver Dysplasia in UC study. ${ }^{27}$ This included prospective surveillance of dysplasia and ploidy status from biopsies

Table 1 Summary of studies evaluating primary sclerosing cholangitis (PSC) as a risk factor for colorectal neoplasia in chronic ulcerative colitis (UC)

\begin{tabular}{|c|c|c|c|c|c|c|}
\hline Study & $\begin{array}{l}\text { UC case group } \\
\text { (No) }\end{array}$ & Centre & End point (No) & $\begin{array}{l}\text { Matched } \\
\text { controls }\end{array}$ & Colectomy rate & $\begin{array}{l}\text { Is PSC a risk } \\
\text { factor? }\end{array}$ \\
\hline Broomé $1992^{14}$ & Dys (17) & Huddinge, Sweden & PSC (5) & Yes & $0 \%$ & Yes \\
\hline D’Haens $1993^{16}$ & Dys (29) & Chicago, USA & Cholestasis/PSC (10) & Yes & $0 \%$ & Yes \\
\hline Broomé $1995^{17}$ & PSC (40) & Huddinge, Sweden & CRC/Dys (15) & Yes & $30 \%$ & Yes \\
\hline Brentnall $1996^{18}$ & PSC (20) & Seattle, USA & Dys (9) & No & $0 \%$ & Yes \\
\hline Leidenius $1997^{19}$ & PSC (45) & Helsinki, Finland & CRC/Dys (13) & Yes & $29 \%$ & Yes \\
\hline Marchesa $1997^{20}$ & PSC (27) & Cleveland, USA & CRC (4)/Dys (14) & Yes & All postop & Yes \\
\hline Shetty $1999^{21}$ & PSC (132) & Cleveland, USA & CRC (17)/Dys (16) & No & $0 \%$ & Yes \\
\hline Harewood $1999^{22}$ & PSC (110) & Mayo Clinic, USA & CRN (35) & No & $\mathrm{n} / \mathrm{s}$ & Yes \\
\hline Choi $1992^{25}$ & PSC (5) & Lahey Clinic, USA & CRC/Dys (2) & No & $\mathrm{n} / \mathrm{s}$ & No \\
\hline Gurbuz $1995^{26}$ & PSC (35) & Johns Hopkins, USA & CRC/Dys (13) & No & $3 \%$ & Unclear \\
\hline Loftus $1996^{23}$ & PSC (143) & Mayo Clinic, USA & CRC (8) & No & $37 \%$ & No \\
\hline Nuako $1998^{24}$ & CRC (171) & Mayo Clinic, USA & PSC (30) & Yes & $14 \%$ & No \\
\hline
\end{tabular}

CRC, colorectal cancer; CRN, colorectal neoplasia; Dys, dysplasia; $\mathrm{n} / \mathrm{s}$, not specified 


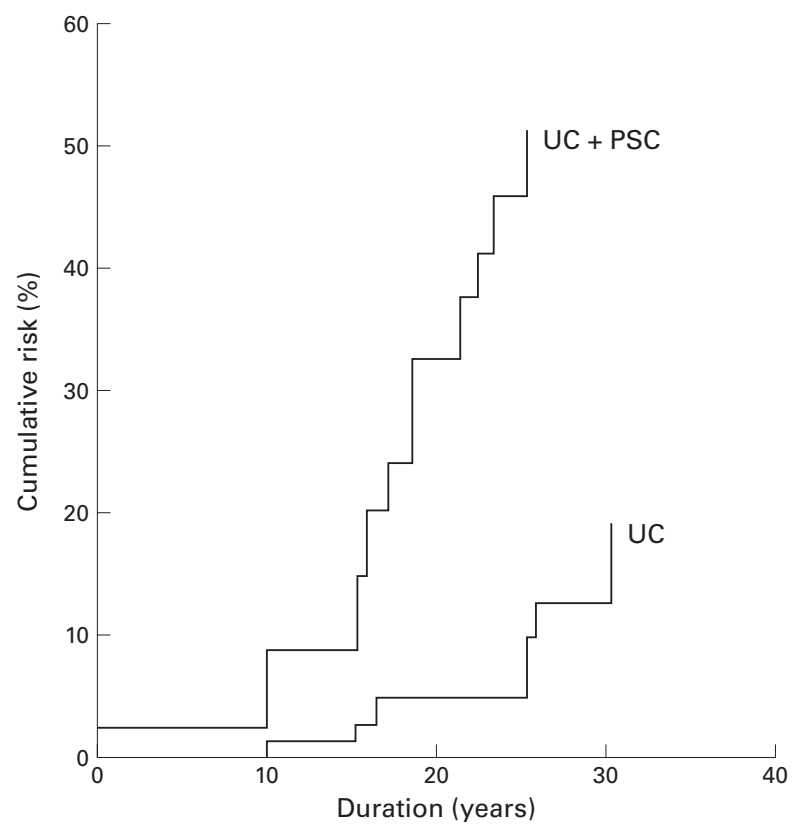

Figure 1 Absolute cumulative risk of developing colorectal neoplasia in patients with ulcerative colitis and primary sclerosing cholangitis (UC+PSC) compared with patients with UC alone $(p<0.001)$ (from Broomé and colleagues ${ }^{17}$ ).

obtained on colonoscopy. DNA aneuploidy was more common in those patients with coexistent PSC than those without. Secondly, multiple synchronous sites of aneuploidy were observed in all those with PSC and abnormal epithelial histology (dysplasia/carcinoma).

These preliminary findings reported in abstract form provide further support for the hypothesis that PSC may be an additional risk factor for colorectal cancer in chronic UC, and that the presence of aneuploidy may be a useful indicator of an increased risk of malignant transformation.

Broomé and colleagues ${ }^{17}$ then provided further evidence to support their initial hypothesis with a case control study intending to assess the absolute cumulative risk of colorectal neoplasia in patients with both UC and PSC against matched controls with UC only. A statistically significant increase in the development of neoplasia was evident in the group with coexistent PSC. A graph of their results is shown in fig 1. Patients with both UC and PSC showed a cumulative risk of developing colorectal neoplasia of $9 \%$ at 10 years, $31 \%$ at 20 years, and $50 \%$ at 25 years after diagnosis of PSC. The corresponding risks for the control group were $2 \%, 5 \%$, and $10 \%$, respectively, representing a fivefold increase in risk at 25 years. A similar risk of neoplasia in the control group to that in recent population based studies of extensive $\mathrm{UC}^{4}$ confirmed that this was a representative control group.

In addition, those patients with both UC and PSC who developed colorectal dysplasia or carcinoma appeared to be at increased risk of developing cholangiocarcinoma. Six patients developed frank carcinoma compared with only one in the control group. ${ }^{17}$

Further positive data were reported by investigators in Seattle. Brentnall and colleagues ${ }^{18}$ reported on a prospective analysis of a group of 20 patients with UC and PSC, and a control group of 25 patients with UC alone. They hoped to evaluate the natural course of the development of colonic dysplasia and carcinoma in both groups. Figure 2 shows a summary of their findings. There was a statistically significant increase in the presence of both dysplasia and aneuploidy in the study group. These patients were five times as likely to develop dysplasia and six times as likely to

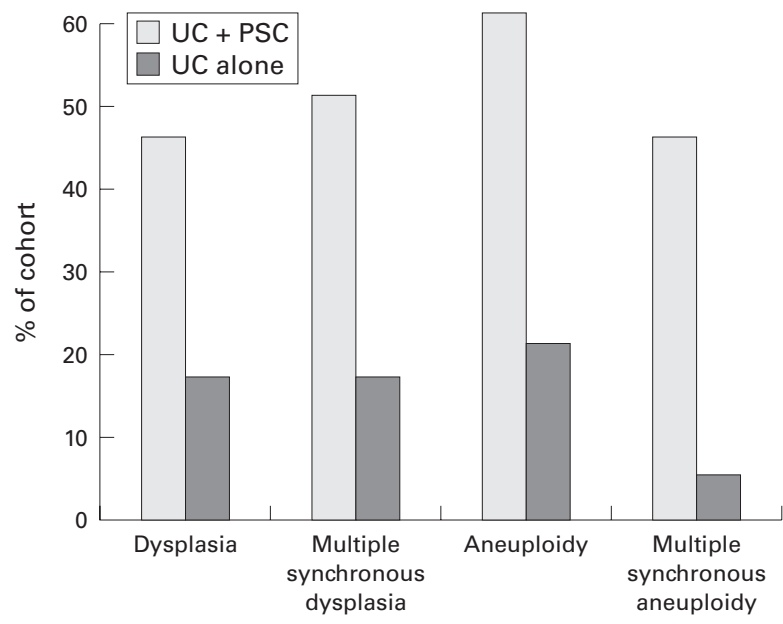

Figure 2 Results of analysis of 20 patients with ulcerative colitis and primary sclerosing cholangitis (UC+PSC) and 25 patients with UC alone with respect to colorectal dysplasia and aneuploidy. (Follow up over a nine year period. ${ }^{18}$ )

develop aneuploidy than the control group with UC alone. After completion of follow up at nine years, $45 \%$ of patients with UC and PSC had been found to have dysplasia compared with $16 \%$ of the control patients with UC alone $(\mathrm{p}=0.002)$

The high prevalence of aneuploidy detected by flow cytometry is particularly noteworthy, supporting other data that UC patients with negative histology but with aneuploidy are more likely to develop colorectal dysplasia than those with normal diploid DNA content. ${ }^{28}$ No difference was apparent between groups in duration of colitis or age of onset.

Kornfeld and colleagues reported a population based study of Swedish patients with an intact colon in UC with coexistent PSC and showed a cumulative risk of cancer of $10 \%, 35 \%$, and $40 \%$ at 10,20 , and 30 years after diagnosis of UC. ${ }^{29}$ In those patients with a prior diagnosis of UC, the risk of developing colorectal cancer 10 years after the diagnosis was $25 \%$. However, the finding that half of the cases were detected prior to diagnosis of PSC led to the suggestion that the association with PSC may not be a causal one.

Investigators from Cleveland conducted a retrospective cohort study comparing 27 patients with both PSC and UC with 1185 patients with UC alone. ${ }^{20}$ All patients had undergone total proctocolectomy between 1983 and the start of analysis. They showed cancer and dysplasia rates that were higher in the group with PSC and UC compared with the group with UC alone. It was also suggested that proximal cancers were more common in UC patients with concomitant PSC compared with patients with UC alone.

Most recently, Shetty et al reported a large retrospective cohort study from Cleveland, evaluating the development of colorectal carcinoma or dysplasia in UC patients with concomitant PSC compared with a control group with UC alone. ${ }^{21}$ This showed that $25 \%$ of the study group went on to develop colorectal carcinoma or dysplasia in comparison with $5.6 \%$ of the control group

THE MAYO CLINIC DATA

A large retrospective cohort comprising 178 patients with PSC from the Mayo Clinic was investigated to determine rates of survival and of development of colorectal cancer. ${ }^{23}$ The risk of colorectal neoplasia was increased in patients with UC and PSC but was not significantly higher than the group with UC alone. On their initial analysis, these authors found no significant increase in the number of cancer cases observed when compared with an expected value, based on a population based cohort from Sweden. ${ }^{4}$ 
A further large retrospective case control study from the Mayo Clinic was then reported, comparing the cumulative prevalence of PSC in UC complicated by carcinoma, compared with a matched group with UC alone. ${ }^{24}$ Again, these investigators concluded that PSC was not associated with an increased risk of colorectal cancer in patients with existing UC. However, as expected, an increased prevalence of PSC was seen with greater duration and extent of colitis.

In discussing these data, the authors suggested that the link between PSC and colorectal cancer may be attributable to PSC acting as a "surrogate marker" for pancolonic and long duration of disease, rather than as an independent risk factor. ${ }^{30}$ Subclinical cases of PSC may have been missed in both groups as well as the premalignant colonic changes such as dysplasia and aneuploidy, which may complicate UC.

These initial reports from the Mayo Clinic led to the suggestion that if PSC is an additional risk factor for neoplasia in UC, its clinical significance is minimal. However, the conclusion drawn from the most recent data from the same centre, available in abstract form only at present, appear to conflict with the initial observations. ${ }^{22}$ Of 110 patients evaluated with PSC, 35 had developed colorectal neoplasia in a median follow up period of 14.7 years. The authors also hypothesise that the separate phenotype of inflammatory bowel disease which is associated with PSC, is distinct from typical UC or Crohn's, and suggest that this may explain the variable prevalence of typical UC in PSC populations.

\section{OTHER STUDIES}

Initially, investigators at the Lahey Clinic ${ }^{25}$ found that PSC had no significant effect on the development of colorectal neoplasia in longstanding UC. However, this conclusion was drawn from a prospective study of patients with longstanding UC, of whom only five had concomitant PSC. In 1995, investigators at Johns Hopkins described a series of 35 patients with UC complicated by PSC. ${ }^{26}$ This study pointed towards a higher than expected rate of dysplasia but no significant increase in colorectal cancers above that expected.

\section{Critical review: difficulties in design and interpretation}

Table 1 summarises the recent studies on the role of PSC as a risk factor for neoplastic transformation in UC. The difficulties in interpreting clinical trials in this situation are highlighted by the fact that they appear to reach conflicting conclusions. We suggest that any of a number of confounding factors may have contributed to the disparate results between studies. These are discussed below.

\section{CHOICE OF CONTROLS}

Selection of an appropriate control group is critical in the interpretation of any study. ${ }^{31}$ For example, Loftus and colleagues $^{23}$ studied a PSC cohort which had been referred for evaluation for liver transplantation. However, they used a population based group (from another country) as the control. It is very likely that a cohort at such a tertiary centre will undergo more surveillance and active intervention in comparison with a population based group. This referral bias alone may have an important effect on the interpretation of the study.

The geographical and temporal disparity between control and study groups in the same study merits consideration. The data set of patients with both UC and PSC tends to be from a later period (1984-1991) than the control data (1919-1983). Again, the medical/surgical management may well have been different in the groups studied. ${ }^{19}$
In the recent study from Cleveland, the group with UC and PSC experienced greater colonic involvement and a longer duration of disease (and were younger at the onset of colitis) than the control group of patients with UC alone. Clearly, these differences may introduce bias into any conclusions made ${ }^{45}$ and serve to emphasise the importance for accurately matched control groups in future studies.

Moreover, the onset of disease is often difficult to define accurately. Colitis associated with PSC is nearly always pancolonic with a mild natural course that rarely leads to colectomy for disease activity. ${ }^{32}$ This is important when comparisons are made against a control UC population as an increased neoplastic risk may merely reflect a longer duration of subclinical colonic disease.

COLECTOMY RATE

A patient group drawn from a single centre may be subjected to an intensified colonoscopic surveillance programme and hence a higher rate of colectomy. Such differences will affect the outcome of the study. In the study by Loftus and colleagues, ${ }^{23} 37 \%$ of the UC-PSC group underwent colectomy, more than one third of which were for dysplasia or cancer prophylaxis.

Observations from population based studies of UC with high colectomy rates ${ }^{33} 34$ showed no significant increase in colorectal neoplasia over that of the general population.

END POINTS OF STUDIES

The end points of the studies vary enormously, for example with Loftus et al using colorectal carcinoma and Brentnall and colleagues ${ }^{18}$ using dysplasia. This is important to consider when comparing the results of trials. However, it has been established that epithelial dysplasia is a premalignant lesion that warrants careful monitoring.

This issue is best illustrated by the data from Seattle. Had Brentnall and colleagues ${ }^{18}$ used colorectal carcinoma as an end point they would have found no difference between the UC-PSC and UC control groups. Conversely, retrospective studies tend to have no set protocol for endoscopic monitoring and so it is likely that a disparity in surveillance intensity will exist between study and control groups.

In many centres, the incidence of dysplasia is higher at the first surveillance colonoscopy. ${ }^{35} \mathrm{~A}$ higher dysplasia rate in the UC-PSC groups might be expected if the corresponding UC control group was already screened and clear of neoplasia.

POWER

With the low prevalence of PSC in the general population, the size of the study group is often very small. Brentnall and colleagues ${ }^{18}$ studied 20 and 25 people in each group, respectively. These small samples have diminished statistical power to detect differences between groups. Similarly, it is difficult to draw firm conclusions from the small series reported by Choi and colleagues. ${ }^{25}$

DRUG THERAPY

The quiescent (or subclinical) nature of the colitis associated with PSC may have led to this group of patients receiving less pharmacotherapy than patients with more troublesome colitis. Hence these patients will not have the benefit of any decrease in colorectal neoplasia which may be associated with such therapy. ${ }^{7}$

ORTHOTOPIC LIVER TRANSPLANTATION

It is important to know whether PSC is a risk factor for colorectal neoplasia as the availability of orthotopic liver transplantation has led to a longer life span for such patients. ${ }^{36}$ Cases of significant colorectal neoplasia have 
been reported within two years of liver transplantation. ${ }^{37} 38$ An observational study of patients with PSC and UC who underwent liver transplantation showed a fourfold increase in the risk of developing neoplasia compared with a control group of similar patients who had not undergone surgery. ${ }^{39}$ It is hypothesised that the intense immunosuppressive therapy given postoperatively may accelerate the dysplasiacarcinoma sequence in patients with UC. However, other investigators have not detected an increased rate of neoplasia or dysplasia in this period. ${ }^{40}$

Recent evidence suggests that UC may be more aggressive after transplantation or even develop de novo, in spite of the use of immunosuppressive drugs. ${ }^{40}$ These data have been reviewed recently. ${ }^{41}$

\section{INTERPRETATION}

Seven of 11 studies reviewed in this paper concluded that PSC had a significant role in the development of neoplasia in chronic UC. The two strongest studies concluding otherwise did not involve matched controls and involved high colectomy rates. Moreover, the most recent data from the Mayo Clinic (albeit only in abstract form at present) appears to contradict the initial observations. It might be ideal to form a meta-analysis of the above data but the heterogeneity of the studies discussed and methodological differences would very likely make any results artificial. The weight of evidence supports the view that PSC is linked with increased neoplasia in chronic UC and that this link does have clinical significance.

\section{Pathogenetic mechanisms}

The mechanisms involved in the development of malignancy in UC are under evaluation. Clinical, epidemiological, and molecular genetic data provide support for the hypothesis that colitis related cancer may have a distinct pathogenesis to sporadic colorectal cancer. ${ }^{42}{ }^{43}$ In comparison with sporadic colorectal cancer, somatic alterations to the p53 tumour suppressor gene occur earlier in colitis related cancers ${ }^{44}$ whereas APC (adenomatous polyposis coli) gene alteration is usually a later event. ${ }^{45}$ The role of microsatellite instability remains uncertain but potentially important. ${ }^{43}$ The direct effect of concomitant PSC has yet to be elucidated.

\section{GENETICS}

The genetic contribution to susceptibility to neoplasia in patients with UC is under evaluation. ${ }^{43} 4647$ Preliminary data have implicated polymorphisms of the mismatch repair genes MLH1 and MSH2 in the pathogenenesis of inflammatory bowel disease and related malignancy. ${ }^{48} 49$ present, germline alterations of cancer susceptibility genes in PSC have not been studied in detail.

\section{BILE SALTS}

It has been suggested that an increased neoplastic risk in the UC-PSC group may be associated with alterations in the bile salt pool due to cholestasis. ${ }^{50}$ Bile acids such as deoxycholic acid and cholic acid may induce gut epithelial proliferation and may also act as tumour promoters in experimental models. ${ }^{51}$ An increased frequency of bile acid receptors in the mucosa in carcinoma compared with normal tissue has been demonstrated. ${ }^{52} \mathrm{~A}$ higher faecal bile acid concentration was found in patients with UC who developed neoplasia compared with those without. ${ }^{53}$ The association of PSC with dysplasia is consistent with a role of colonic bile acids in tumorigenesis in patients with UC. Moreover, this theory may underlie any increased incidence of proximal colorectal cancers in UC complicated by PSC.
FOLATE DEFICIENCY

Patients with UC are often folate deficient as sulphasalazine is a competitive inhibitor of folate absorption. Folate supplementation was associated with a $62 \%$ reduction in the incidence of neoplasia in patients with pancolonic UC compared with placebo. ${ }^{54}$ However, the hypothesis that sulphasalazine related folate deficiency contributes to the development of malignancy cannot be reconciled with the preliminary epidemiological data which raise the possibility that maintenance therapy in UC may be associated with reduced carcinogenesis. ${ }^{78}$

\section{Conclusions}

The weight of evidence suggests an increased risk of colonic neoplasia in patients with chronic UC who have concomitant PSC, although the mechanism is unclear. This increased risk has implications on the physician and patient in terms of screening. If a group of patients who are at an increased risk of developing colorectal carcinoma can be identified, they must be monitored and treated accordingly.

Although there is no consensus at present, Sandborn and colleagues $^{30}$ suggested annual surveillance colonoscopy from the diagnosis of PSC in all patients with concomitant UC. Those patients with PSC who do not exhibit signs of UC should undergo an initial colonoscopy to look for the development of subclinical disease, ${ }^{26}$ with periodic reevaluation.

The issue of UC in the setting of liver transplantation for PSC is posing many new questions. On current evidence, prophylactic colectomy post-transplantation does not appear to be necessary but again annual colonoscopy has been recommended for surveillance. ${ }^{39}$

A large prospective case control study involving patients matched for age, onset of colitis, duration of UC, and transplantation status is required to help determine the magnitude of the increased risk of colorectal neoplasia conferred by the presence of concomitant PSC and may also provide new insight into disease pathogenesis.

H JAYARAM

J SATSANGI

R W G CHAPMAN

Gastroenterology Unit, Radcliffe Infirmary,

Oxford OX2 6HG,

j.satsangi@ed.ac.ukUK

Correspondence to: Professor J Satsangi, Gastrointestinal Unit, University of Edinburgh, Western General Hospital, Edinburgh EH4 2XU, UK.

1 Bargen JA. Chronic ulcerative colitis associated with malignant disease. Arch Surg 1928;17:561-76.

2 Gilat T, Fireman Z, Grossman A, et al. Colorectal cancer in patients with ulcerative colitis. A population study in central Israel. Gastroenterology 1988;94:870-7.

3 Broström O, Löfberg R, Nordenvall B, et al. The risk of colorectal cancer in ulcerative colitis. An epidemiologic study. Scand $\mathcal{F}$ Gastroenterol 1987;22: 1193-9.

4 Ekbom A, Helmick C, Zack M, et al. Ulcerative colitis and colorectal cancer. A population-based study. N Engl f Med 1990;323:1228-33.

5 Gyde SN, Prior P, Allan RN, et al. Colorectal cancer in ulcerative colitis: a cohort study of primary referrals from three centres. Gut 1988;29:206-17.

6 Riddell RH, Goldman H, Ransohoff DF, et al. Dysplasia in inflammatory bowel disease: standardized classification with provisional clinical applications. Hum Pathol 1983;14:931-68.

7 Pinczowski D, Ekbom A, Baron J, et al. Risk factors for colorectal cancer in patients with ulcerative colitis: a case-control study. Gastroenterology 1994; 107:117-20

8 Moody GA, Jayanthi V, Probert CS, et al. Long-term therapy with sulphasalazine protects against colorectal cancer in ulcerative colitis: a retrospective study of colorectal cancer risk and compliance with treatment in Leicestershire. Eur $\mathcal{F}$ Gastroenterol Hepatol 1996;8:1179-83.

9 Chapman RW, Arborgh BA, Rhodes JM, et al. Primary sclerosing cholangitis: a review of its clinical features, cholangiography, and hepatic histology. Gut 1980;21:870-7.

10 Rosen CB, Nagorney DM. Cholangiocarcinoma complicating primary sclerosing cholangitis. Semin Liver Dis 1991;11:26-30.

11 Schrumpf E, Elgjo K, Fausa O, et al. Sclerosing cholangitis in ulcerative colitis. Scand f Gastroenterol 1980;15:689-97.

12 Olsson R, Danielsson A, Järnerot G, et al. Prevalence of primary sclerosing cholangitis in patients with ulcerative colitis. Gastroenterology 1991; 100(5Pt1):1319-23. 
13 Balasubramaniam K, Wiesner RH, LaRusso NF. Primary sclerosing cholangitis with normal serum alkaline phosphatase activity. Gastroenterology 1988:95:1395-8.

14 Broomé U, Lindberg G, Löfberg R. Primary sclerosing cholangitis in ulcerative colitis - a risk factor for the development of dysplasia and DNA aneuploidy? Gastroenterology 1992;102:1877-80.

15 Broomé U, Chapman RW. Ulcerative colitis: sclerosing cholangitis today, cancer tomorrow? Gut 1997;41:571-2.

16 D'Haens GR, Lashner BA, Hanauer SB. Pericholangitis and sclerosing cholangitis are risk factors for dysplasia and cancer in ulcerative colitis. $\mathrm{Am}$ f Gastroenterol 1993;88:1174-8.

17 Broomé U, Löfberg R, Veress B, et al. Primary sclerosing cholangitis and ulcerative colitis: evidence for increased neoplastic potential. Hepatology 1995;22:1404-8.

18 Brentnall TA, Haggitt RC, Rabinovitch PS, et al. Risk and natural history of colonic neoplasia in patients with primary sclerosing cholangitis and ulcerative colitis. Gastroenterology 1996;110:331-8

19 Leidenius MH, Farkkila MA, Karkkainen P, et al. Colorectal dysplasia and carcinoma in patients with ulcerative colitis and primary sclerosing cholancarcinoma in patients with ulcerative colitis
gitis. Scand $\mathcal{f}$ Gastroenterol 1997;32:706-11.

20 Marchesa P, Lashner BA, Lavery IC, et al. The risk of cancer and dysplasia among ulcerative colitis patients with primary sclerosing cholangitis. $A m \mathcal{F}$ Gastroenterol 1997:92:1285-8.

21 Shetty K, Rybicki L, Brzezinski A, et al. The risk for cancer or dysplasia in ulcerative colitis patients with primary sclerosing cholangitis. Am $\mathcal{F}$ Gastroenterol 1999;94:1643-9.

22 Harewood GC, Loftus EV, Tremaine WJ, et al. "PSC-IBD": a unique forum of inflammatory bowel disease associated with primary sclerosing cholangitis. Gastroenterology 1999;116:G3178.

23 Loftus EV Jr, Sandborn WJ, Tremaine WJ, et al. Risk of colorectal neoplasia in patients with primary sclerosing cholangitis. Gastroenterology 1996;110: 432-40

24 Nuako KW, Ahlquist DA, Sandborn WJ, et al. Primary sclerosing cholangitis and colorectal carcinoma in patients with chronic ulcerative colitis: a case-control study. Cancer 1998;82:822-6.

25 Choi PM, Nugent FW, Rossi RL. Relationship between colorectal neoplasia and primary sclerosing cholangitis in ulcerative colitis. Gastroenterology and primary scler

26 Gurbuz AK, Giardiello FM, Bayless TM. Colorectal neoplasia in patients with ulcerative colitis and primary sclerosing cholangitis. Dis Colon Rectum with ulcerative colit $1995 ; 38: 37-41$.

27 Ahnen DJ, McHugh JBD, Arsenault LL, et al. Does PSC increase the risk of colon cancer in patients with chronic ulcerative colitis? Gastroenterology 1993;104:A692

28 Rubin CE, Haggitt RC, Burmer GC, et al. DNA aneuploidy in colonic biopsies predicts future development of dysplasia in ulcerative colitis. Gastroenterology 1992;103:1611-20.

29 Kornfeld D, Ekbom A, Ihre T. Is there an excess risk for colorectal cancer in patients with ulcerative colitis and concomitant primary sclerosing cholangitis? A population based study. Gut 1997;41:522-5.

30 Sandborn WJ, Loftus EV Jr, Ahlquist DA. Association of primary sclerosing cholangitis and colorectal cancer in patients with ulcerative colitis: is it true and does it matter? Gastroenterology 1998;115:236-7.

31 Ahnen DJ. Controlled clinical trials: the controls are the key. Gastroenterology 1996;110:628-30.

32 Lundqvist $\mathrm{K}$, Broomé U. Differences in colonic disease activity in patients with ulcerative colitis with and without primary sclerosing cholangitis: a case control study. Dis Colon Rectum 1997;40:451-6.
33 Stonnington CM, Phillips SF, Zinsmeister AR, et al. Prognosis of chronic ulcerative colitis in a community. Gut 1987;28:1261-6.

34 Langholz E, Munkholm P, Davidsen M, et al. Colorectal cancer risk and mortality in patients with ulcerative colitis. Gastroenterology 1992;103: 1444-51.

35 Lennard-Jones JE. Is colonoscopic cancer surveillance in ulcerative colitis essential for every patient? Eur F Cancer 1995;31A(7-8):1178-82.

36 Marsh JW Jr, Iwatsuki S, Makowka L, et al. Orthotopic liver transplantation for primary sclerosing cholangitis. Ann Surg 1988;207:21-5.

37 Higashi H, Yanaga K, Marsh JW, et al. Development of colon cancer after liver transplantation for primary sclerosing cholangitis associated with ulcerative colitis. Hepatology 1990;11:477-80.

38 Bleday R, Lee E, Jessurun J, et al. Increased risk of early colorectal neoplasms after hepatic transplant in patients with inflammatory bowel disease. Dis Colon Rectum 1993;36:908-12.

39 Loftus EV Jr, Aguilar HI, Sandborn WJ, et al. Risk of colorectal neoplasia in patients with primary sclerosing cholangitis and ulcerative colitis following orthotopic liver transplantation. Hepatology 1998;27:685-90.

40 Papatheodoridis GV, Hamilton M, Mistry PK, et al. Ulcerative colitis has an aggressive course after orthotopic liver transplantation for primary sclerosing cholangitis. Gut 1998;43:639-44.

41 Ala A, Burroughs A, Hamilton M. Inflammatory bowel disease after orthotopic liver transplantation for primary sclerosing cholangitis. Inflamm Bowel Dis Monitor 2000;1:104-8.

42 Itzkowitz SH. Inflammatory bowel disease and cancer. Gastroenterol Clin North Am 1997;26:129-39.

43 Itzkowitz SH. Microsatellite instability in colitis-associated colorectal cancer. Gut 2000;46:304-5.

44 Yin J, Harpaz N, Tong Y, et al. p53 point mutations in dysplastic and cancerous ulcerative colitis lesions. Gastroenterology 1993;104:1633-9.

45 Redston MS, Papadopoulos N, Caldas C, et al. Common occurrence of $\mathrm{APC}$ and K-ras gene mutations in the spectrum of colitis-associated neoplasias. Gastroenterology 1995;108:383-92.

46 Sandborn WJ. Inflammatory bowel disease and hereditary nonpolyposis colorectal cancer: is there a genetic link? Gastroenterology 1998;114:608-9.

47 Satsangi J, Marshall SE, Welsh KI, et al. Cancer susceptibility genes in ulcerative colitis. Gut 2000;46 (suppl II):A6.

48 Pokorny RM, Hofmeister A, Galandiuk S, et al. Crohn's disease and ulcerative colitis are associated with the DNA repair gene MLH1. Ann Surg 1997;225:718-23.

49 Brentnall TA, Rubin CE, Crispin DA, et al. A germline substitution in the human MSH2 gene is associated with high-grade dysplasia and cancer in ulcerative colitis. Gastroenterology 1995;109:151-5.

50 Nagengast FM, Grubben MJ, van Munster IP. Role of bile acids in colorectal carcinogenesis. Eur f Cancer 1995;31A(7-8):1067-70.

51 Suzuki K, Bruce WR. Increase by deoxycholic acid of the colonic nuclear damage induced by known carcinogens in C57BL/6J mice. $\mathcal{F}$ Natl Cancer Inst 1986;76:1129-32.

52 Summerton J, Flynn M, Cooke T, et al. Bile acid receptors in colorectal cancer. Br f Surg 1983;70:549-51.

53 Hill MJ, Melville DM, Lennard-Jones JE, et al. Faecal bile acids, dysplasia, and carcinoma in ulcerative colitis. Lancet 1987;2:185-6.

54 Lashner BA, Heidenreich PA, Su GL, et al. Effect of folate supplementation on the incidence of dysplasia and cancer in chronic ulcerative colitis. A case-control study. Gastroenterology 1989;97:255-9. 